A. Pramesh Rao, G. Swarup and Gopal-Krishna, eds.

\title{
Radio Continuum Surveys of the Galaxy and Galaxies
}

\author{
Richard Wielebinski \\ Max-Planck-Institut für Radioastronomie, Auf dem Hügel 69, 53121 \\ Bonn, Germany
}

\begin{abstract}
Radio sky surveys give us basic information about the origin of the radio emission from the Galaxy. By mapping the sky at several radio frequencies a separation of the thermal and non-thermal emission components is possible. The major part of the low-frequency radio emission comes from the synchrotron process, the braking of relativistic electrons in magnetic fields. By mapping the linear polarization at several frequencies (required for the correction of the Faraday rotation) the orientation of the magnetic fields in the emitting regions can be deduced. Older all-sky surveys at 30,150 and $408 \mathrm{MHz}$ have now been supplemented by new observations of the Galaxy at 45 and $1420 \mathrm{MHz}$. These surveys, in addition to being important as tracers of the morphology of the magnetic fields in the Galaxy, are also required to correct for the 'foreground' features in cosmological studies of the COBE data and the PLANCK surveys in the future. Studies of the Galaxy in polarization have been made some years ago indicating high percentage of linear polarization in various directions. More recent work with good angular resolution has shown spectacular polarized intensity structures in selected regions. Lowfrequency data with good angular resolution are urgently required for the interpretation of these features.
\end{abstract}

Observations of nearby galaxies in radio continuum (both total power and polarized intensity) have given us the possibility to study magnetic fields in objects at known distances. Polarization observations of nearby galaxies have confirmed the existence of regular magnetic fields in practically every object so far studied. Originally data were obtained from large single-dish telescopes, notably from Effelsberg and Parkes. These data were greatly enhanced by the addition of higher resolution components from the VLA and ATCA respectively. These results indicate surprisingly homogeneous magnetic fields in most galaxies. High angular resolution observations with the GMRT at lower radio frequencies will add a new dimension to the data on galaxies.

\section{Introduction}

All-sky surveys in radio continuum require the combination of observations from both the northern and southern hemispheres and are relatively rare. The first such all-sky map was presented by Dröge \& Priester (1956) at $200 \mathrm{MHz}$ and showed the dominant Galactic plane emission, the North Polar Spur and the 
maxima in the directions of Cygnus $\mathrm{X}$ and Vela $\mathrm{X}$, the tangential directions of the local spiral arm. The $150 \mathrm{MHz}$ map of Landecker \& Wielebinski (1970) had better angular resolution and allowed the delineation of additional spurs that were interpreted to be nearby supernova remnants. Also the regions of lowest galactic emission were seen more clearly in the southern sky putting a low upper limit on any halo emission. A combination of surveys at $30 \mathrm{MHz}$ by Cane (1978) supported the suggestion of the north-south anomaly. The all-sky survey at $408 \mathrm{MHz}$ (Haslam et al. 1982) became a standard for many years. New surveys with careful calibration of absolute temperatures at 45 and $1420 \mathrm{MHz}$ have now been completed (Maeda et al. 1999; Testori et al. 2000) giving us important spectral data.

Linearly polarized radio emission, which is the definite signature of magnetic fields, was first detected by Wielebinski et al. (1962) and Westerhout et al. (1962) at $408 \mathrm{MHz}$. A survey of the northern sky was published by Wielebinski \& Shakeshaft (1964). The southern sky was mapped by Mathewson \& Milne (1965). These early observations showed that the linearly polarized component was high, with polarization percentage $P>10 \%$ in some directions. It became clear that both Faraday depolarization and beam depolarization were affecting the observations. In several steps the observing frequency went up to $1400 \mathrm{MHz}$ (e.g. Brouw \& Spoelstra 1976) giving a clearer picture of the distribution of polarized Galactic emission. However the 'internal' observer's view of the Galaxy complicated any interpretation.

The main effort in recent years was devoted to observing external galaxies in polarization. The first observations of radio polarization in the galaxy M51 were made by Mathewson et al. (1972). Polarization maps of the Andromeda nebula (M31) by Beck et al. $(1978 ; 1980)$ followed. By now most of the nearby galaxies have been mapped showing regular magnetic fields (e.g. Wielebinski \& Krause 1993; Beck et al. 1996). An interesting result is the great similarity in the observed magnetic fields for galaxies of very different morphological type. The general deduction, from recent observations of galaxies at $1400 \mathrm{MHz}$, which were a result of the combination of the Effelsberg and VLA data, is that polarized intensity is a good tracer of ordered magnetic fields. Progress in this field of research awaits high angular resolution observations of polarized intensity with the GMRT at lower radio frequencies.

\section{A Medium-Latitude Survey of the Galaxy}

The early polarization surveys of the Galaxy showed that the maxima of polarization occur above and below the Galactic plane. More recently Duncan et al. (1999) re-analyzed a $2.7 \mathrm{GHz}$ Galactic plane survey in polarization and showed that the polarized intensity increases to higher Galactic latitudes. All these results suggest that there is substantial Faraday depolarization along the Galactic plane and that the detailed magnetic field structure can be delineated only by mapping the linear polarization in higher Galactic latitudes. Building on these results a medium-latitude survey was started with the Effelsberg radio telescope at $1.4 \mathrm{GHz}$ by Uyaniker et al. (1998; 1999). In particular, the calibration aspects had to be carefully considered since no absolute values of the Stokes parameters $(\mathrm{Q}, \mathrm{U})$ are usually measured, only relative values, relative 
to a defined baseline. These baselines can be partly deduced from published, absolutely calibrated, low-resolution polarization survey data.
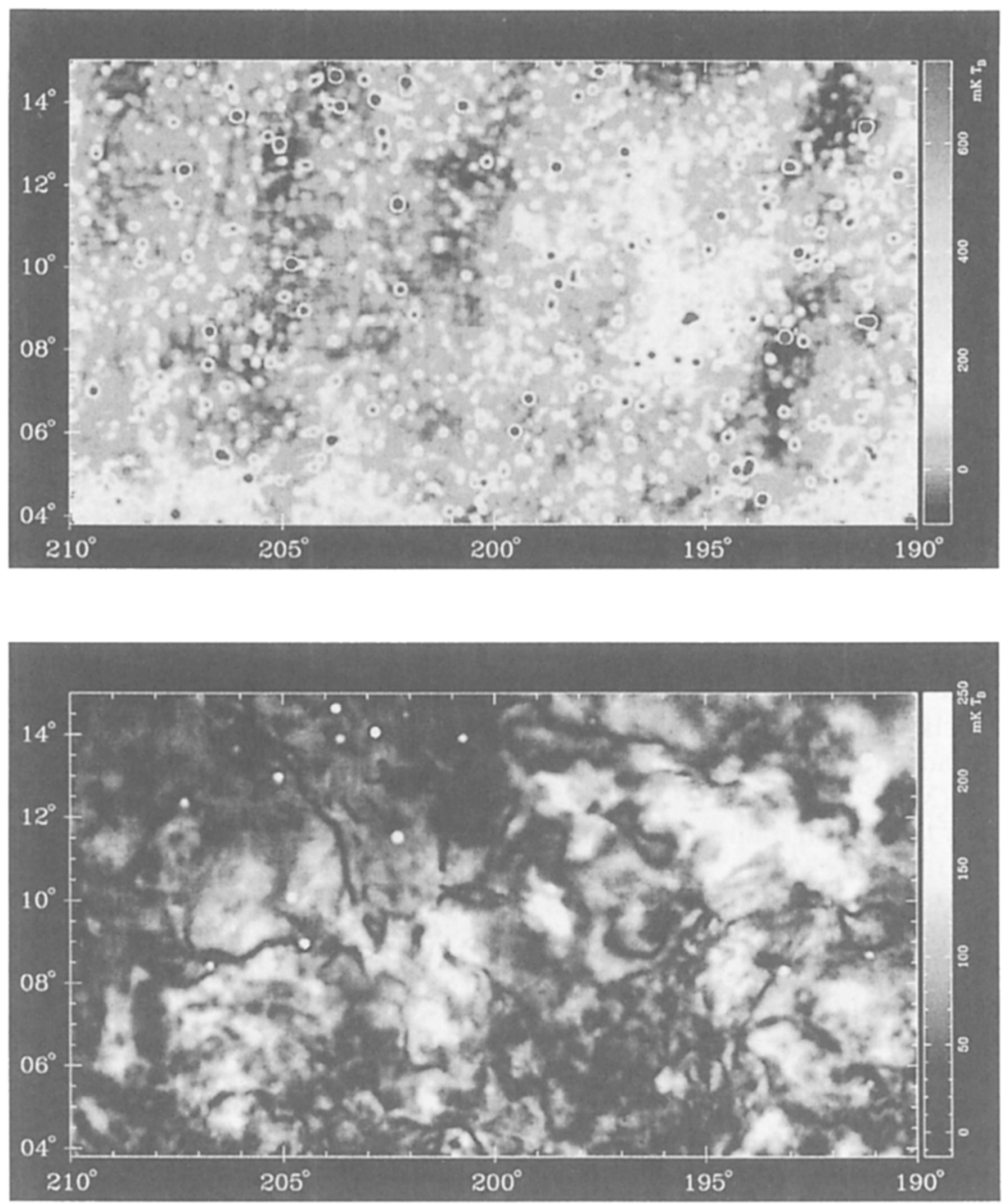

Figure 1. Total intensity (above) and polarized intensity (below) of the region centered on $l=200^{\circ}, b=+10^{\circ}$ from Uyaniker et al. (1999).

Observations of several large Galactic regions have been published in Uyaniker et al. (1999). Figure 1 shows the maps for the anomalous anti-center region centered on $l=200^{\circ}, b=+10^{\circ}$. This is a region of sky where a rather flat spectral index has been observed, suggestive of thermal material above the plane, resulting in high Faraday rotation measures. The sudden nulls in polarized intensity, which appear as filamentary structures, could be a result of the differential Faraday rotation passing through the characteristic nulls (e.g. Sokoloff et al. 1998) and/or due to filamentary structure of the thermal matter. 
Observations at higher radio frequencies are in progress to help understand these bizarre structures.

Small-scale polarization structures have been observed at $325 \mathrm{MHz}$ in the diffuse Galactic emission by Wieringa et al. (1993). The apparent polarization brightness was $\sim 4 \mathrm{~K}$ and some prominent filaments $\sim 4^{\circ}$ long were observed. Recent observations with the $1.4 \mathrm{GHz}$ system in Effelsberg (W. Reich, private communication) confirmed many of the features observed in the $l=171^{\circ}, b=$ $18^{\circ}$ field by Wieringa. The filamentary structures which are on the $\sim 1$ arcmin scale can be seen, but with a reduced intensity, in view of the 9 arcmin beam. However two larger areas of linear polarization are seen in the maps at both frequencies. This result and studies of other areas at high Galactic latitudes suggest the presence of considerable linear polarization everywhere.

\section{Radio Continuum Surveys of Galaxies}

Several surveys of the total radio continuum intensity of galaxies have appeared in the literature (e.g. Condon 1987; Niklas et al. 1995) in addition to numerous maps of individual galaxies. Polarized radio emission in a galaxy was first detected in M51 by Mathewson et al. (1972). Polarized emission in M31 was mapped by Beck et al. $(1978,1980)$. Both these observations implied the presence of homogeneous magnetic fields on scales of kiloparsecs in spiral galaxies. Since that time the consequent use of the Effelsberg radio telescope and later of the VLA, the Parkes telescope and the ATCA gave us a huge database on magnetic fields in galaxies. In particular, the combination of the single-dish observations with interferometer data gave us maps with good angular resolution at frequencies as low as $1.4 \mathrm{GHz}$ (e.g Beck et al. 1998). The overall picture that we get from these observations is that well-ordered magnetic fields exist in most galaxies, of different morphological type. Figure 2 shows the magnetic fields and the polarized intensity of the galaxy NGC 6946 (Beck \& Hoernes 1996). Homogeneous magnetic fields are observed also in irregular and dwarf galaxies. Observations of edge-on galaxies give us the additional information that the magnetic fields are concentrated to the disks of galaxies (e.g. Dumke et al. 1995). In some galaxies, like in NGC 4631, a vertical magnetic field component was observed in the nucleus. Vertical magnetic fields are also seen in the Galactic Center (e.g. Seiradakis et al. 1989). This leads to the suggestion that the morphology of polarized intensity in NGC 4631 and our Galaxy is similar.

\section{Discussion}

The magnetic fields that are observed in galaxies are surprisingly homogeneous on scales of many kiloparsecs. This seems to be in direct contrast to the structures of Galactic emission which have been detected in the Effelsberg mediumlatitude survey. Viable interpretation has been advanced for the large-scale magnetic fields in the context of the multi-mode dynamo theory (e.g. Wielebinski \& Krause 1993; Beck et al. 1996). Considering the angular resolution of the different observations we must suspect that the large-scale magnetic fields could be caused by the amplification of a seed field in the galactic rotation while the small-scale magnetic fields are a result of local activity. In fact the 

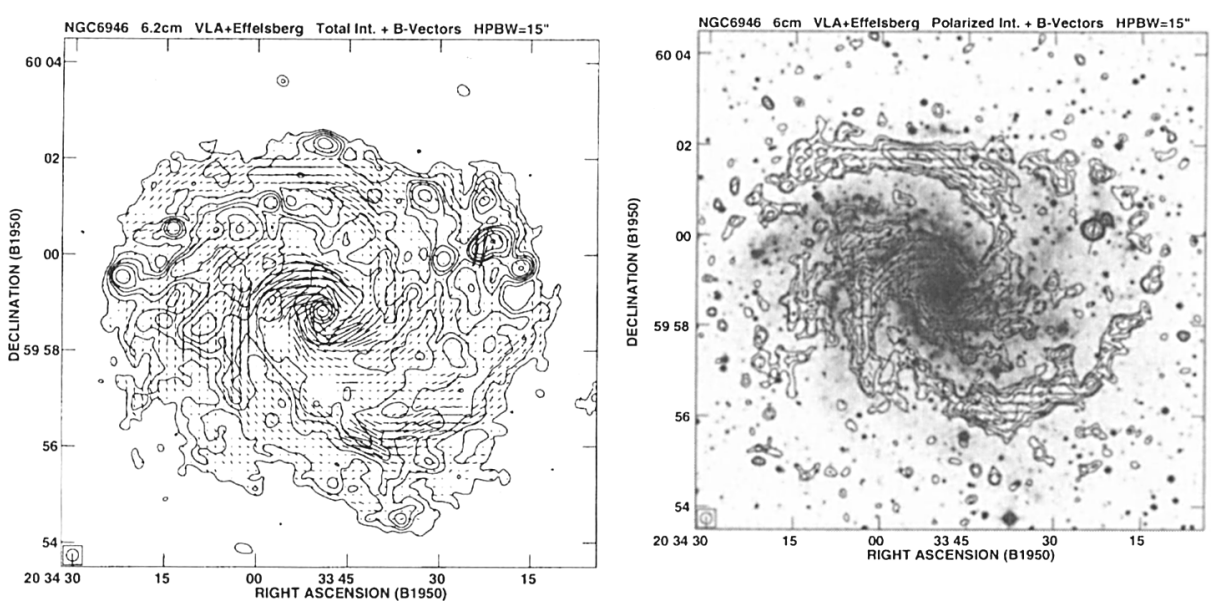

Figure 2. The galaxy NGC 6946. Total intensity with superposed ' $B$ ' vectors (left) and polarized intensity (right). Courtesy R. Beck, MPIfR

dynamo action requires seed fields which could be generated by local activity (e.g. supernovae, stellar winds, local turbulence, fast moving objects, etc.). This suggests that magnetic fields are a basic constituent of the interstellar medium, a component that has been badly neglected in the past. Since low-frequency, high-resolution radio continuum maps of galaxies are a direct indicator of the magnetic field morphology the GMRT will add new and important knowledge to this subject.

Acknowledgments. I would like to thank the 'continuum group' at the MPIfR, in particular W. Reich, B. Uyaniker, E. Fürst and R. Beck, who made these beautiful radio continuum maps possible.

\section{References}

Beck, R., Berkhuijsen, E.M., \& Wielebinski, R. 1978 A\&A, 68, L27

Beck, R., Berkhuijsen, E.M., \& Wielebinski, R. 1980, Nature, 283, 272

Beck, R., \& Hoernes, P. 1996, Nature, 379, 47

Beck, R., Brandenburg, A., Moss, D. et al. 1996, ARA\&A, 34, 153

Beck, R., Berkhuijsen, E.M., \& Hoernes, P. 1998, A\&AS, 129, 329

Brouw W.N., \& Spoelstra, T.A.Th. 1976, A\&AS, 26, 129

Cane, H.V. 1978, Aust. J. Phys., 31, 561

Condon, J.J. 1987, ApJS, 65, 485

Dröge, F., \& Priester, W. 1956, Zeitschrift f. Phys., 40, 236

Duncan, A.R., Reich, P., Reich, W., \& Fürst, E. 1999, A\&A, 350, 447

Dumke, M., Krause, M., Wielebinski, R., \& Klein, U. 1995, A\&A, 302, 691

Haslam, C.G.T., Salter, C.J., Stoffel, H., \& Wilson, W.E. 1982, A\&AS, 47, 1 
Landecker, T.L., \& Wielebinski, R. 1970, Aust. J. Phys. Suppl. No. 16 Maeda, K., Alvarez, H., Aparici, J. et al. 1999, A\&AS, 140, 145

Mathewson D.S., \& Milne D.K. 1965, Aust. J. Phys., 18, 635

Niklas, S., Klein, U., Braine, J., \& Wielebinski, R. 1995, A\&AS, 114, 21

Seiradakis, J.H., Reich, W., Wielebinski, R. et al. 1989, A\&AS, 81, 291

Sokoloff, D.D., Bykov, A.A., Shukurov, A. et al. 1998, MNRAS, 299, 189

Testori, J.C., Reich, P. et al. 2000 (in preparation)

Uyaniker, B., Fürst, E., Reich, W. et al. 1998, A\&AS, 132, 401

Uyaniker, B., Fürst, E., Reich, W. et al. 1999, A\&AS, 138, 31

Westerhout, G., Seeger, Ch.L., Brouw, W.N. et al. 1962, Bull. Astr. Inst. Ned., 16,187

Wielebinski, R., \& Krause, F. 1993 A\&AR, 4, 449

Wielebinski, R., \& Shakeshaft, J.R. 1964, MNRAS, 268, 19

Wielebinski, R., Shakeshaft, J.R., \& Pauliny-Toth, I.I.K. 1962, Observatory, 82, 158

Wieringa, M.H., de Bruyn, A.G., Jansen, D. et al. 1993, A\&A, 268, 215 